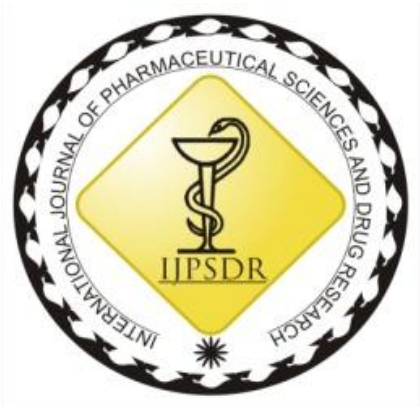

\author{
RESEARCH ARTICLE
}

ISSN: 0975-248X

CODEN (USA): IJPSPP

$($ (c) $)$ EY-NC-SA

\title{
The Effectiveness of Selective Stem Cell Placement on Gait Performance in Patients with Multiple Sclerosis: a Phase I Clinical Trial
}

\author{
David S. Cohen ${ }^{1}$, Henry Broeska ${ }^{2 *}$ \\ ${ }^{1}$ School of Medicine, University of Massachusetts, Boston, USA \\ ${ }^{2}$ Saddleback College, California, USA
}

Copyright (C) 2019 David S. Cohen et al. This is an open access article distributed under the terms of the Creative Commons AttributionNonCommercial-ShareAlike 4.0 International License which allows others to remix, tweak, and build upon the work non-commercially, as long as the author is credited and the new creations are licensed under the identical terms.

\begin{abstract}
Multiple sclerosis (MS) is a chronic and oftentimes disabling disease of the central nervous system. It affects almost 1 million people living in the United States and about 2.5 million people globally. Though the cause is not fully understood, MS occurs through an inflammatory process in the brain. This inflammation results in the destruction of the myelin sheath surrounding the nerves that enable conduction of nerve impulses within the brain and spinal cord. To overcome symptoms, MS patients are usually prescribed disease modifying drug therapies or immunosuppressive drugs for prolonged periods resulting in only temporary clinical improvement. In a large subset of patients, disease progression is measured by the continuing deterioration of physical ability. One of the most reliable measures of disease progression in MS is gait performance. Previous studies have demonstrated that clinically significant gait deviations occur at all stages of MS that involve walking. This report describes a Phase I clinical trial of a novel targeted delivery technique, selectively placing autologous bone marrow mononuclear stem cells into the central vein of multiple sclerosis patients, with post-operative functional changes measured by gait performance. To determine the functional benefit of selective stem cell placement, the timed 25 -foot walk was administered to $31 \mathrm{MS}$ patients before and after therapy. The time and number of steps taken to complete the test significantly decreased post-intervention. Currently, disease-modifying and immunosuppressive drugs are the only approved treatments for MS. Comparative trials between disease-modifying and immunosuppressive drugs measure benefit as a slowing of multiple sclerosis manifestations, not an improvement. This trial demonstrated an immediate global improvement in the level of disability as measured by the timed 25-foot walk. This suggests that recovery of function in MS patients is possible. Where pharmaceutical agents only mitigate symptoms and potentially slow disease progression, selective stem cell placement of autologous bone marrow mononuclear stem cells should be considered as a possible treatment for MS due to measured improvement in post-therapy functional ability. Further research is required to validate our findings.
\end{abstract}

Keywords: Multiple Sclerosis - Stem Cells - Trial - Gait - Bone Marrow.

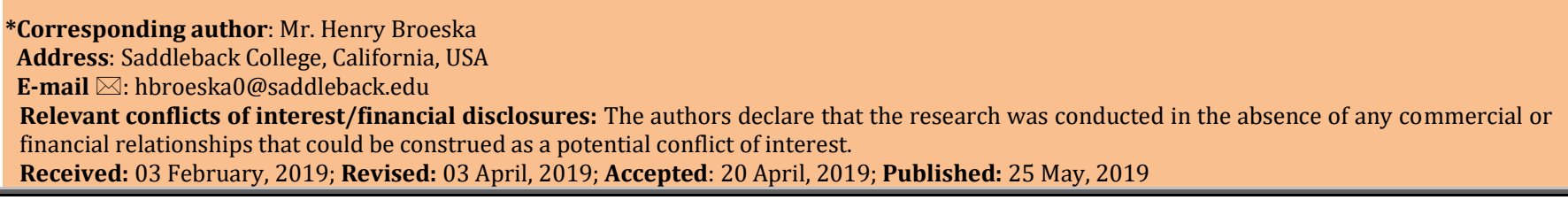




\section{INTRODUCTION}

Multiple sclerosis (MS) is a chronic, multifactorial disease of the central nervous system (CNS), and one of the most common causes of permanent disability in young adults. [1] It is an epigenetically-mediated autoimmune response involving inflammatory mechanisms that eventually lead to nerve degeneration and unpredictable risks of grey matter atrophy, lesion spread and consequent cognitive impairment, physical disability, and lower quality of life. ${ }^{[1-2]}$

The incidence rate is approximately 3.6 MS cases per 100,000 person-years globally. [3] The US has around 12,000 new diagnoses of MS per year. [4] In terms of prognosis, MS patients' life expectancy can be 5-10 years shorter than that of the general population. [5] Financially, MS is a major burden on patients and the healthcare industry at large, with MS therapies costing $\sim \$ 60,000$ annually, and increasing significantly each year. This is more problematic in the U.S., where prices of medications are up to three times higher than in other countries. [6]

It has been shown that women are more susceptible to many more autoimmune diseases than men. [7] In MS, the male to female sex ratio is approximately 1:3, thereby making gender one of the top predictors. [8] Age of onset is typically between 20 to 40 years. Some studies demonstrate that $3-5 \%$ of the MS population experience symptoms before the age of 16. [9-10] Early onset of sensory disturbances, optic neuritis, and brainstem dysfunction are more prevalent in women around the age of puberty, suggesting that sex hormones may play a role in MS. [11] Historically, the earliest pathological post-mortem investigations observed vascular anomalies in brain structures. This led to the hypothesis that the pathogenesis of MS could be a blood-brain barrier disorder leading to progressive neurological impairment. [12-15] Blood-brain barrier (BBB) dysfunction is now considered to be a characteristic feature of MS noted early in the disease's course, [16-18] but the critical matter as to the cause of primary inflammation and the spread of lesions remains an open question. [15]

Current treatments for MS are only partially effective due to the inability to affect the cause of defective remyelinating/regenerating mechanisms, resulting in chronic, accumulating disability and irreversible axonal/neuronal damage. $[1,17]$ Hence, in order to improve treatment outcome in MS, innovative approaches are required for immune regulation different than non-selective immunosuppression.

The use of bone marrow mononuclear stem cells (BMMNSCs) in clinical studies, inclusive of hematopoietic and mesenchymal cells, which have endothelialregenerative and immunomodulatory properties, have been found to be safe, well tolerated, and counteract nerve damage to promote neurogenesis. Indeed, neurorestorative therapies have been demonstrated in experimental models that use stem cells from various sources, including BM-MSCs for neurological diseases such as Huntington's disease. [19-20] Although significant long-term benefit including recovery of functional ability still remains to be seen in clinical trials, there is considerable optimism that stem cell therapy provides a new frontier in the treatment of MS as well.

Autologous BM-MNSCs could be a highly effective therapy in MS for several important reasons related to their natural characteristics. 1) Stem cells can be safely re-infused from autologous sources (patients themselves being potential donors) without the need for immunosuppression to prevent rejection [21]; 2) they can be derived from adult BM, and if necessary, cultured in vitro and expanded in number; 3 ) stem cells have the multi-potential ability to self-renew and to differentiate into other cell types including neurons and glial cells in response to their immediate environment or signals from other cells; 4) they have exceptional immunoregulatory properties; 5) they are less disposed to genetic aberrations resulting in a small risk for induction of malignancies in contrast to other sources of stem cells; 6) they secrete neurotrophic factors that stimulate both developing and mature endogenous neural cells in the CNS.

The purpose of this Phase I clinical trial was to assess the safety of the treatment in addition to changes in the functional ability of MS patients who received transplantation of autologous BM-MNSCs by way of targeted, minimally-invasive selective stem cell placement (SSCP).

\section{MATERIALS AND METHODS}

\section{Ethical statement}

Formal ethical approval was granted by the Independent Ethics Committee, Pune (India). This committee is under governance and oversight of the Drugs Controller General India (DCGI), the equivalent of the U.S. Food and Drug Administration (FDA) in India. All procedures and potential risks were explained to each research study participant. Written informed consent was obtained for each participant prior to data collection. All of the methods were conducted in accordance with the relevant regulations, guidelines for research, and the Declaration of Helsinki. All research study participants were under the direct care of a full team of health care professionals, including a neurosurgeon, an interventional radiologist, a hematologist, and a team of nurses and attendants for the duration of the study.

\section{Selective Stem Cell Placement}

All research study participants underwent a novel selective stem cell placement (SSCP), which included four distinct procedures. All participants remained resident in the hospital for a period of 13 to 15 days. Additional post-operative management required study participants to be kept under the supervision of the hospital's Neurological Surgery Director.

Bone marrow aspiration (BMA) 
Participants were taken to an in-hospital catheterization laboratory (Cathlab). A hematologist aspirated 150-200 $\mathrm{ml}$ of bone marrow (depending on the patient's size and weight) from the iliac crest under local anesthesia using a Rosenthal large bore aspiration needle. The aspirate was added to ethylenediaminetetraacetic acid (EDTA) anticoagulant $(1.50 \pm 0.25 \mathrm{mg} / \mathrm{ml})$ to preserve morphology, and then immediately processed by washing, using phosphate-buffered saline (1\% PBS), and re-suspending in heparinized normal saline (final volume: $5 \mathrm{ml}$ ). Bone marrow stem cells were isolated through centrifugation to obtain a concentrated phase containing mononuclear cells (MNC). The plasma was separated and discarded from the mononuclear fraction.

\section{Balloon venoplasty and stem cell infusion procedures} Post-BMA, the participants remained in the Cathlab and were prepared for venoplasty and stem cell infusion. These were administered using a minimally invasive proprietary therapeutic algorithm for which the nomenclature, 'selective stem cell placement' or 'SSCP' (patent pending), has been developed. Study participants received concentrated autologous bone marrow-derived stem cells by proximal transfusion to the cranial veins utilizing fluoroscopic guidance and modified angioplasty techniques, concurrent with selective stem cell placement. Immediately after the procedure, participants were taken to the recovery room where hospital staff monitored post-anesthesia and post-procedural care. Participants remained in recovery for approximately one hour while they were observed for any adverse events or pain. They were taken back to their hospital rooms once it was determined they were stable enough for discontinuance of acute care/observation but under continuous monitoring and supervision of the hospital's Neurological Surgery Director.

\section{Aspiration of cerebrospinal fluid (CSF)}

Four days following the venoplasty procedure, the participants underwent aspiration of CSF. Aspiration of CSF was performed via a spinal tap (L3-L4 interspace). After local anesthesia was administered to both the surface and deeper tissues, fluid was withdrawn through a spinal needle (26 gauge) via lumbar puncture with the participant on his or her side in the left lateral position. The amount of CSF appropriate to the size and weight of the participant (as determined by the neurologist) was withdrawn $(80-150 \mathrm{ml})$ into $20 \mathrm{ml}$ syringes. The CSF was then used for immediate reinfusion during the intrathecal stem cell infusion procedure.

\section{Autologous transplant through CSF}

Participants received a stem cell infusion immediately post-CSF aspiration through the same spinal needle. The already positioned 26 gauge spinal needle was fitted with a double lumen adaptor. One lumen was used for injection of approximately $1 \times 10^{6}$ autologous stem cells, while the other was used for the syringes containing the pre-collected CSF. Both solutions were concurrently injected over a two to three-minute period. The spinal needle was left in place for an additional minute post-infusion to prevent leakage and then removed.

\section{Measures}

Researchers have previously found that walking speed (WS) as a measurement parameter can be used to monitor changes in patients with MS [22-23], owing to its reliability and sensitivity as a measure of functional ability. [24-26] Specifically, the timed 25-foot walk (T25FW) has been used as a valid and reliable technique for a wide range of MS populations. [22, 27] In this study, the T25FW was used as per standardized instructions. [28] The walking test was performed immediately pre-intervention (pre-SSCP) and at 10 days post-intervention (post-SSCP) on the same walking track. Participants were given the choice to use walking assistive aides as required. All subjects were timed and videotaped. The number of steps and time taken (in seconds) to complete the test was recorded for each participant. Scores were collected pre- and posttherapy and the differences calculated.

Statistical analysis

The skewness and kurtosis tests were used for testing the normal distribution of continuous variables. Wilcoxon signed-rank and Mann-Whitney U tests were used to non-parametric variables for related and unrelated samples, respectively. Spearman's rho correlation was used to evaluate the correlation between variables. All data were analyzed using the Statistical Package for Social Sciences (SPSS) (version $20)$; tests were two-tailed with $a=0.001, P$-value of $<$ 0.05 was considered as statistically significant.

\section{RESULTS}

\section{Research Study Participants}

In this study $(N=31)$, the patient cohort was $77 \%$ female, and 23\% male; similar to the rate at which MS occurs in males and females in the global population. [8] Ages ranged from 26 to 64 years $(M=48.0, S D=10.5)$. The age distribution was slightly negatively skewed ($.53)$, indicating that most participants were in the older age range. Specifically, $12.9 \%$ were 39 years or younger, $16.1 \%$ were between the ages of 35 years and $44,38.7 \%$ were between the ages of 45 and 54 years, and $32.3 \%$ were 55 years or older.

The MS classifications were $77.4 \%$ with a progressive form of MS (41.9\% PPMS and 35.5\% SPMS), and the remaining $22.6 \%$ had RRMS. Participants also reported the number of years since they received their diagnosis of MS: $29 \%$ received their diagnosis 5 years (or less) prior to the time of data collection, $16 \%$ received their diagnosis 6-10 years ago, 39\% received theirs diagnosis 11-20 years ago, and 16\% were diagnosed with MS 21 years (or more) prior to the time of data collection. Participants' Expanded Disability Status Scale (EDSS [29]) scores ranged from 3.5 to 7.0 (from a possible range of 1-10, with higher scores reflecting more severe 
disability), with mean and median values of 5.58 and 6 values, respectively.

No adverse effects were observed while completing the T25FW. Despite wide-ranging abilities, 29 (93.5\%) patients demonstrated improvement in completing the T25FW (Figure 1).

On average, participants took $22.7 \%$ less time and $17.4 \%$ fewer steps to complete the T25FW post-SSCP. More than half of the participants used walking assistance (e.g., rollators, canes) for their walk test $(58.1 \%)$, while the remaining participants were able/chose to walk with no assistance (41.9\%). MannWhitney $U$ tests indicated that the distribution of the percentages of change in time $\left(U_{\text {Time }}=53.0, p=0.14\right)$ and steps $\left(U_{\text {Steps }}=61.0, p=0.28\right)$ were not significant across sex - i.e., there was no gender difference in improvement in walking post-SSCP. A series of Spearman's rho correlations demonstrated that the percentages of change in $\mathrm{T} 25 \mathrm{FW}$ completion were slightly lower for participants who were older $\left(r_{s_{-} \text {Time }}=\right.$ $\left.-0.17, p=0.36 ; r_{s_{-} \text {Steps }}=-0.24, p=0.19\right)$ and had their diagnosis for a longer time $\left(r_{s_{-} \text {Time }}=-0.15, p=0.42\right.$; $r_{s_{-} \text {Steps }}=-0.17, p=0.36$ ), however these results were not significant. Similarly, no significant differences in change were observed for MS type $\left(r_{s_{-} \text {Time }}=-0.16, p=\right.$ $\left.0.39 ; r_{s_{-} \text {Steps }}=0.01, p=0.95\right)$.

On average, post-SSCP $(M=31$ seconds, $S D=34.48)$ time scores were lower than pre-SSCP scores $(M=41.5$ seconds, $S D=45.42)$. Similar results were found for number of steps taken: on average, participants used fewer steps to complete the T25FW post-SSCP $(M=$ $18.41, S D=6.07)$, as compared to pre-SSCP $(M=22.45$, $S D=6.7)$. Additionally, pre and post-SSCP time $(r=$
$0.98, p<0.001)$ and step scores $(r=0.87, p<0.001)$ have a strong positive correlation, indicating that participants with high scores prior to intervention also had high scores post-intervention.

The Wilcoxon signed-rank tests indicated that the median post-SSCP time score $(M d n=16.45)$ was significantly lower than that of the median pre-SSCP time score $(M d n=24.57), Z=-4.70(p<0.001)$. Similarly, the median post-SSCP step score $(M d n=17)$ was significantly lower than that of the pre-SSCP step score $(M d n=22), Z=-4.65(p<0.001)$. The effect sizes of the SSCP treatment were also calculated and were medium $(r=0.6)$ for both measures. Therefore, the SSCP procedure had a medium effect on both the amount of time and number of steps taken to complete the T25FW test.

No adverse effects were observed in this Phase I clinical trial, significant or otherwise, in a two-year postprocedure follow-up.

\section{DISCUSSION}

Currently, there is no therapy for either relapsingremitting or the progressive phases of MS that results in a significant reversal of disability. Conventional treatment methods are primarily targeted towards symptom relief rather than the pathophysiology of the disease. [2] Our objectives were to demonstrate that selective intravenous autologous stem cell implantation can be safely performed with a response that results in improved functional ability. A review of approved drug therapies reveals the inadequacies of conventional treatments.

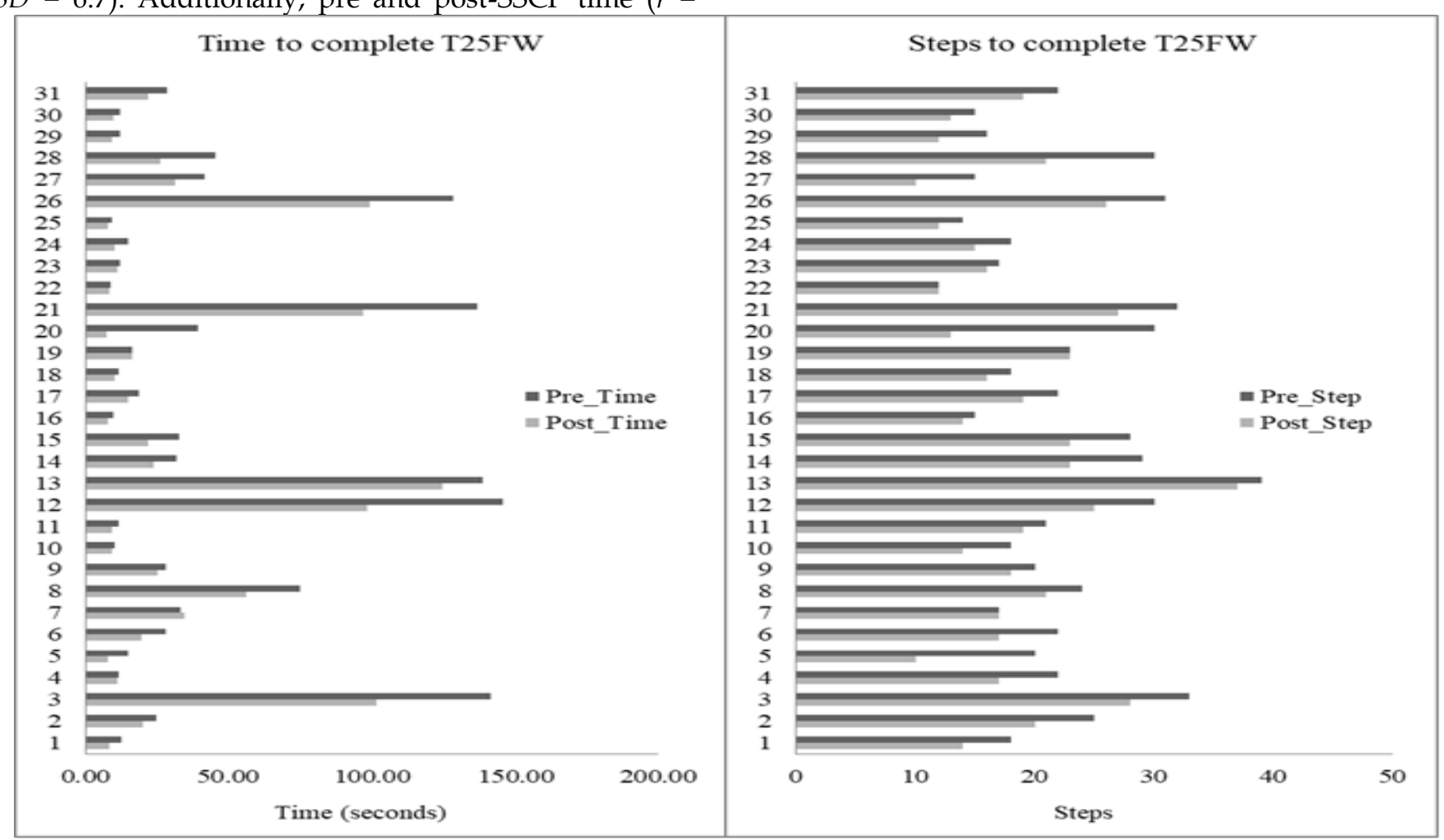

Fig. 1: This figure illustrates that almost all participants demonstrated improvement in walking the 25-foot distance under controlled conditions post-SSCP. Only two patients did not show any improvement. 
In 1993, Interferon Beta-1a became the first pharmacological approved for the treatment of the relapsing/remitting course of the disease. Known as a disease-modifying therapy (DMT), claims have been made by the manufacturers that Interferon Beta-1a is modestly effective at reducing the number of attacks and the accumulation of brain lesions in RRMS. Unfortunately, interferon Beta-1a does not provide any immediate or obvious benefit and can have adverse side effects for MS patients. Despite manufacturersponsored data showing some efficacy for DMT, there is no claim of enduring benefit. The degenerative characteristics of MS mean that if symptoms can be affected at all, they can only be managed temporarily with DMT. As a result, many MS patients choose not to take these drugs citing various reasons ${ }^{[30]}$. Chief among their concerns are that many patients do not believe they are receiving enough benefit for the expense and risk involved for its continuous, long-term use. In addition, these drugs are not meant to treat the progressive phases of the disease, only the relapsing/remitting course.

More recent biological medications such as Ocrelizumab, approved by the Food and Drug Administration for treatment of both courses of MS, relapsing/remitting and progressive. Ocrelizumab, under the trade name of 'Ocrevus,' is a humanized antiCD20 monoclonal antibody. Ocrevus targets CD20 marker on B lymphocytes and hence is classified as an immunosuppressive drug. [31] It was initially believed that treatment based upon twice-annual parenteral administration of the monoclonal antibody drug might offer a better-tolerated treatment option compared to the multiple monthly injection regime of DMT. However, an adverse effect of immunosuppressive drugs is immuno-deficiency since they act nonselectively, resulting in increased vulnerability to infections as well as reduced cancer immunosurveillance. [32-33] Researchers have reported on 12 and 24-week disability progression versus placebo with mixed results. [34] A critical review of manufacturersponsored research concludes that the long-term results of a similar drug (Natalizumab) produced progressive multi-focal leukoencephalopathy (PML). Over a longer term than the Ocrevus study reported on, this resulted in significant adverse events including recorded deaths and severe disabilities induced by the drug rather than the participants' disease. [35]

Given that stem cell therapies have made significant headway in MS research, our goal was to determine whether selective stem cell placement (SSCP), a novel therapeutic procedure, can be used as an effective treatment for MS patients. [2,36] Instead of a systemic approach, this novel protocol is aimed at implanting the patient's own stem cells selectively at the site of injury. Infusion of autologous cells by way of selective venous catheterization seeks to take advantage of the cells' natural ability to differentiate by allowing the immediate micro-environment to determine chemotactic response. [37] Studies demonstrate that autologous stem cells have the ability to differentiate into a variety of tissues [38] and can infiltrate the bloodbrain barrier. This method of delivery ensures a higher concentration of repair cells than otherwise would be the case directed toward the target area to resolve pathology specific to the known plaque-vein relationship in MS. ${ }^{[15,39]}$ Additionally, there is reduced risk in transplanting cells from one location in the patient's body to another, particularly in light of the fact that these same stem cells circulate continuously in lower concentrations in peripheral blood.

The primary end-point was a change in disability measured by differences in the T25FW pre- and posttherapy. Walk speed is one of the most sensitive measures for detecting clinical and subclinical disability in multiple sclerosis. [22] Thirty-one research study participants were asked to complete the timed 25-foot walk, before and after receiving SSCP. Although there was slightly more improvement in completing the T25FW among participants who were younger and who had their diagnosis for a shorter period of time, age, gender, MS type, and years since diagnosis was not significantly associated with the degree of improvement in completing the T25FW. Thus, there is a reason to believe that SSCP may have the potential to benefit all MS patients. No adverse effects were noted, which echoes the findings from previous studies using autologous bone marrow mononuclear stem cell (BMMNSC) transplants [2,36], including transplants through the spinal canal. [40] This provides evidence for SSCP's safety and tolerability as a treatment and rationale for utilizing the same procedure with a larger sample since primary endpoints were achieved and significant differences were found between baseline pre-therapy and follow-up post-therapy measurements.

Nonetheless, the findings of this study should be interpreted in light of some caveats. Given that our primary interest was to assess functional ability and that it was not feasible to assess demyelination lesions through MRI, more rigorous research is needed to confirm our findings on the neurophysiological level. In other words, the efficacy of SSCP as a potential breakthrough therapy for people with MS cannot be determined without confirming neuroregeneration (e.g., remyelination) with larger sample sizes and longitudinal studies. As the failure of the BBB is a crucial early occurrence in MS pathogenesis, any therapy that overcomes $\mathrm{BBB}$ dysfunction and reestablishes functional ability without the risk of collateral long-term immunotoxic effects may well offer a new paradigm as a first-line treatment for MS patients. [41] Given the clinically significant improvement in participant performance in this study, it is plausible that the BM-MNSCs replaced and repaired degenerated endothelial tissue associated with dysfunction of the blood-brain barrier (through an 
angiogenic mechanism) [42], counteracted nerve damage and promoted rapid neurogenesis (e.g., by propelling oligodendrocyte progenitor cells [OPCs]), which is consistent with previous research. [2,36,43-46]

Double-blind, placebo-controlled clinical trials are needed to support the efficacy of any new treatment. Alternatively, SSCP could be compared to conventional therapies - i.e., patients who wish to continue conventional treatment can be assessed for improvement in functionality and neuroregeneration compared to patients who wish to undergo SSCP. It is also important to note that patients who have been minimally ambulatory for many years (much like some of the participants in this study) can develop severe bone deformities, muscle loss, and permanent contracture of tendons that cannot easily be overcome by the interruption of the disease itself. Therefore, it is possible that this novel treatment may be more beneficial for individuals who are in less progressive phases of the disease, or those who have not developed persistent mechanical, connective and soft-tissue abnormalities caused by degenerative disc disease and nerve root compression as a result of persistent or progressive MS. This is a confounding factor that should be considered when interpreting the efficacy of potential new lines of treatment for MS that are aimed at restoring any level of functional ability.

Each patient served as his or her own control, many having failed to respond to standard pharmacological treatments over the course of their disease. After a single stem cell infusion in the method described, this preliminary study demonstrated that recovery of functional ability is possible for people with MS, regardless of age, gender, MS type, or years since diagnosis. Measured differences in walk test parameters pre- and post-SSCP may be explained by the novel targeted, site-specific method of treatment. SSCP deserves further investigation with larger samples, longer follow-up times, and testing of other outcome functions such as dexterity, cognition, and MRI. If efficacy is confirmed by further study, making this approach available in health care settings has the potential to dramatically increase the number of patients who could benefit from this therapy as a firstline treatment. This article is a prologue to our next publication where we evaluate patient outcomes over a 24-month follow-up.

\section{ACKNOWLEDGMENTS}

We would like to thank our research study participants and their families for helping us to launch this study.

\section{REFERENCES}

1. Larochelle C, Uphaus T, Prat A, Zipp F. Secondary progression in multiple sclerosis: neuronal exhaustion or distinct pathology? Trends Neurosci. 2016;39(5):325-39.

2. Chahine NA, Wehbe T, Rashed J, Hilal R, Elias N. Autologous bone marrow derived stem cells for the treatment of multiple sclerosis. International journal of stem cells. 2016;9(2):207.
3. Alonso A, Hernán MA. Temporal trends in the incidence of multiple sclerosis: a systematic review. Neurology. 2008;71(2):129-35

4. Hirtz D, Thurman D, Gwinn-Hardy K, Mohamed M, Chaudhuri A, Zalutsky R. How common are the "common" neurologic disorders? Neurology. 2007;68(5):326-37.

5. Compston A, Coles A. Seminar Multiple sclerosis. Lancet. 2008;372:1502-17.

6. Hartung DM, Bourdette DN, Ahmed SM, Whitham RH. The cost of multiple sclerosis drugs in the US and the pharmaceutical industry: too big to fail? Neurology. 2015;84(21):2185-92.

7. Ngo ST, Steyn FJ, McCombe PA. Gender differences in autoimmune disease. Front Neuroendocrinol. 2014;35(3):34769.

8. Magistrale G, Pisani V, Argento O, Incerti CC, Bozzali M, Cadavid D, et al. Validation of the World Health Organization Disability Assessment Schedule II (WHODASII) in patients with multiple sclerosis. Multiple Sclerosis Journal. 2015;21(4):448-56.

9. Ghezzi A, Deplano V, Faroni J, Grasso M, Liguori M, Marrosu G, et al. Multiple sclerosis in childhood: clinical features of 149 cases. Multiple Sclerosis Journal. 1997;3(1):43 6.

10. Ruggieri M, Polizzi A, Pavone L, Grimaldi LM. Multiple sclerosis in children under 6 years of age. Neurology. 1999;53(3):478-.

11. Boiko A, Vorobeychik G, Paty D, Devonshire V, Sadovnick D. Early onset multiple sclerosis: a longitudinal study. Neurology. 2002;59(7):1006-10.

12. Noseworthy J. Lucchinetti C Rodriguez M, et. al. Medical progress: multiple sclerosis NEJM. 2000;343:938-52.

13. Tettey P, Simpson Jr S, Taylor BV, van der Mei IA. Vascular comorbidities in the onset and progression of multiple sclerosis. J Neurol Sci. 2014;347(1-2):23-33.

14. Christiansen CF, Christensen S, Farkas DK, Miret M, Sørensen HT, Pedersen L. Risk of arterial cardiovascular diseases in patients with multiple sclerosis: a populationbased cohort study. Neuroepidemiology. 2010;35(4):267-74.

15. Schelling F. Multiple sclerosis: the image and its message. The meaning of the classic lesion forms www multiplesclerosis-abc org. 2007.

16. Minagar A, Alexander JS. Blood-brain barrier disruption in multiple sclerosis. Multiple Sclerosis Journal. 2003;9(6):540-9.

17. Larochelle C, Alvarez JI, Prat A. How do immune cells overcome the blood-brain barrier in multiple sclerosis? FEBS Lett. 2011;585(23):3770-80.

18. Troletti CD, de Goede P, Kamermans A, de Vries HE. Molecular alterations of the blood-brain barrier under inflammatory conditions: the role of endothelial to mesenchymal transition. Biochimica et Biophysica Acta (BBA)-Molecular Basis of Disease. 2016;1862(3):452-60.

19. Kerkis I, Haddad MS, Valverde CW, Glosman S. Neural and mesenchymal stem cells in animal models of Huntington's disease: Past experiences and future challenges. Stem Cell Res Ther. 2015; 14;6:232.

20. Rossignol J, Fink KD, Crane AT, Davis KK, Bombard MC, Clerc S, Bavar AM, Lowrance SA, Song C, et al. Reductions in behavioral deficits and neuropathology in the R6/2 mouse model of Huntington's disease following transplantation of bone-marrow-derived mesenchymal stem cells is dependent on passage number. Stem Cell Res Ther. 2015; 19;6:9.

21. Ullah I, Subbarao RB, Rho GJ. Human mesenchymal stem cells-current trends and future prospective. Biosci Rep. 2015;35(2):e00191.

22. Middleton A, Fritz SL, Lusardi M. Walking speed: the functional vital sign. Journal of aging and physical activity. 2015;23(2):314-22.

23. Nogueira LAC, dos Santos LT, Sabino PG, Alvarenga RMP, Santos Thuler LC. Factors for lower walking speed in persons with multiple sclerosis. Multiple sclerosis international. $2013 ; 2013$. 
24. Adell E, Wehmhörner S, Rydwik E. The test-retest reliability of 10 meters maximal walking speed in older people living in a residential care unit. J Geriatr Phys Ther. 2013;36(2):74-7.

25. Rydwik E, Bergland A, Forsen L, Frändin K. Investigation into the reliability and validity of the measurement of elderly people's clinical walking speed: a systematic review. Physiother Theory Pract. 2012;28(3):238-56.

26. Verghese J, Wang C, Holtzer R. Relationship of clinic-based gait speed measurement to limitations in community-based activities in older adults. Arch Phys Med Rehabil. 2011;92(5):844-6.

27. Behrens J, Pfüller C, Mansow-Model S, Otte K, Paul F, Brandt AU. Using perceptive computing in multiple sclerosis-the Short Maximum Speed Walk test. J Neuroeng Rehabil. 2014;11(1):89.

28. Cutter GR, Baier ML, Rudick RA, Cookfair DL, Fischer JS, Petkau J, et al. Development of a multiple sclerosis functional composite as a clinical trial outcome measure. Brain. 1999;122(5):871-82.

29. Kurtzke JF. Rating neurologic impairment in multiple sclerosis: an expanded disability status scale (EDSS). Neurology. 1983;33(11):1444-.

30. Johnson KL, Kuehn CM, Yorkston KM, Kraft GH, Klasner E, Amtmann D. Patient perspectives on disease-modifying therapy in multiple sclerosis. International Journal of MS Care. 2006;8(1):11-8.

31. McGinley MP, Moss BP, Cohen JA. Safety of monoclonal antibodies for the treatment of multiple sclerosis. Expert Opin Drug Saf. 2017;16(1):89-100.

32. Makinodan T, SANTOS GW, Quinn R. Immunosuppressive drugs. Pharmacol Rev. 1970;22(2):189-247.

33. Vial T, Descotes J. Immunosuppressive drugs and cancer. Toxicology. 2003;185(3):229-40.

34. Kappos L, Li D, Calabresi PA, O'Connor P, Bar-Or A, Barkhof $\mathrm{F}$, et al. Ocrelizumab in relapsing-remitting multiple sclerosis: a phase 2, randomised, placebo-controlled, multicentre trial. The Lancet. 2011;378(9805):1779-87.

35. Chaudhuri A. Ocrelizumab in multiple sclerosis: risks and benefits. The Lancet. 2012;379(9822):1196-7.
36. Trounson A. New perspectives in human stem cell therapeutic research. BMC Med. 2009;7(1):29.

37. Wright DE BE, Wagers AJ, Butcher EC, Weissman IL. Hematopoietic stem cells are uniquely selective in their migratory response to chemokines. J Exp Med. 2002.

38. Le Blanc K, Ringden O. Immunomodulation by mesenchymal stem cells and clinical experience. J Intern Med. 2007;262(5):509-25.

39. Schelling F S. Damaging venous reflux into the skull or spine: relevance to multiple sclerosis. Med Hypotheses. 1986.

40. Oraee-Yazdani S, Hafizi M, Atashi A, Ashrafi F, Seddighi A, Hashemi S, et al. Co-transplantation of autologous bone marrow mesenchymal stem cells and Schwann cells through cerebral spinal fluid for the treatment of patients with chronic spinal cord injury: safety and possible outcome. Spinal Cord. 2016;54(2):102.

41. Kamphuis W, Derada Troletti C, Reijerkerk A, A Romero I, E de Vries $H$. The blood-brain barrier in multiple sclerosis: microRNAs as key regulators. CNS \& Neurological Disorders-Drug Targets (Formerly Current Drug TargetsCNS \& Neurological Disorders). 2015;14(2):157-67.

42. Susienka MJ, Medici D. Vascular endothelium as a novel source of stem cells for bioengineering. Biomatter. 2013;3(3):e24647.

43. Alvarez-Dolado M, Pardal R, Garcia-Verdugo JM, Fike JR, Lee HO, Pfeffer K, et al. Fusion of bone-marrow-derived cells with Purkinje neurons, cardiomyocytes and hepatocytes. Nature. 2003;425(6961):968.

44. Karussis D, Kassis I, Kurkalli BGS, Slavin S. Immunomodulation and neuroprotection with mesenchymal bone marrow stem cells (MSCs): a proposed treatment for multiple sclerosis and other neuroimmunological/ neurodegenerative diseases. J Neurol Sci. 2008;265(1-2):131-5.

45. Tropel P, Platet N, Platel JC, Noël D, Albrieux M, Benabid $\mathrm{AL}$, et al. Functional neuronal differentiation of bone marrow-derived mesenchymal stem cells. Stem Cells. 2006;24(12):2868-76.

46. Yang J, Rostami A, Zhang G-X. Cellular remyelinating therapy in multiple sclerosis. J Neurol Sci. 2009;276(1-2):1-5.

HOW TO CITE THIS ARTICLE: Cohen DS, Broeska H. The Effectiveness of Selective Stem Cell Placement on Gait Performance in Patients with Multiple Sclerosis: a Phase I Clinical Trial. Int. J. Pharm. Sci. Drug Res. 2019; 11(3): 71-77. DOI: 10.25004/IJPSDR.2019.110301 